\title{
Evaluación de técnicas de pretratamiento en buchón de agua (Eichhornia crassipes) para la producción de bioetanol
}

\author{
Karen Ospino, Elkin Gómez y Luis Rios* \\ Procesos Químicos Industriales, Departamento de Ingeniería Química, Facultad de Ingeniería, Universidad de Antioquia \\ U de A, Calle 70 No. 52-21, Medellín, Colombia. (correo-e: ingenierakov@gmail.com; elkina.gomez@udea.edu.co; \\ luis.rios@udea.edu.co)
}

${ }^{*}$ Autor a quien debe ser dirigida la correspondencia.

Recibido Mar. 13, 2019; Aceptado Abr. 30, 2019; Versión final Jul. 29, 2019, Publicado Feb. 2020

\begin{abstract}
Resumen
Se evaluaron cuatro pretratamientos del Buchón de Agua (Eichhornia crassipes) para su transformación en azúcares y etanol. El Buchón de Agua es una planta acuática de rápido crecimiento que representa un serio problema de invasiones por lo que se propone su transformación en etanol, teniendo en cuenta la inminente escasez de combustibles fósiles a nivel mundial. En esta selección preliminar el Buchón de Agua exhibió características constitutivas de tipo químico, que lo convierten en una materia prima promisoria para producción de etanol, obteniéndose, rendimientos de 17,15 g/l, 18,10 g/l, 17,73 g/l y 4,53 g/l con los pretratamientos remojo con amoniaco, delignificación alcalina, pretratamiento con ácido diluido y peróxido alcalino, respectivamente. Finalmente, se seleccionaron los pretratamientos con ácido diluido y delignificación alcalina para ser utilizados con mayor profundidad mediante la aplicación de un diseño experimental, obteniéndose un rendimiento de etanol de 190,35 I/ton biomasa seca alimentada para el pretratamiento ácido diluido y 197,33 I/ton biomasa seca alimentada para el pretratamiento delignificación alcalina, a partir de la fracción sólida.
\end{abstract}

Palabras clave: etanol; Buchón de Agua; biomasa; pretratamiento; biocombustible; delignificación.

\section{Evaluation of pretreatment techniques in water hyacinth (Eichhornia crassipes) for the production of bioethanol}

\begin{abstract}
Water Hyacynth (Eichhornia crassipes) is a fast growing aquatic plant that represents a serious problem of invasion, so its transformation into ethanol is proposed as a solution, taking into account the imminent scarcity of fossil fuels worldwide and the panorama of opportunity that is lived in the world due to the legislation on biofuels. For this, four different pretreatments were evaluated taking into account production of sugars and final ethanol yield to select the most promising. In this preliminary selection, the Water Hyacinth exhibited chemical type compositional characteristics that make it a promising raw material for ethanol production, obtaining yields of $17.15 \mathrm{~g} / \mathrm{l}, 18.10 \mathrm{~g} / \mathrm{l}, 17.73 \mathrm{~g} / \mathrm{l}$ and $4,53 \mathrm{~g} / \mathrm{l}$ with the pretreatments aqueous ammonia soaking, alkaline delignification, pretreatment with diluted acid and alkaline peroxide, respectively. Finally, the pretreatments with diluted acid and alkaline delignification were selected to be studied more deeply through the application of an experimental design, obtaining an ethanol yield (better design conditions). $190.35 \mathrm{l} /$ ton dry biomass fed to the diluted acid pretreatment and $197.33 \mathrm{l} /$ ton dry biomass fed to the alkaline delignification pretreatment, from the solid fraction.
\end{abstract}

Keywords: ethanol; Water Hyacinth; biomass; pretreatment; biofuel; delignification. 


\section{INTRODUCCIÓN}

En Colombia se está presentando una problemática en los embalses de Empresas Públicas de Medellín EPM (Río Grande, Porce II y Guatapé), que actualmente están afectados por la proliferación excesiva de Buchón de Agua (EPM, 2009). Para el año 2009, se removieron más de 81 mil toneladas de Buchón de Agua en base seca. Como alternativa para la producción de combustibles se plantea el Buchón de Agua (Eichhornia crassipes). En India, China, Tailandia, Malasia se reportan trabajos para aprovechar y resolver problemáticas asociadas a su crecimiento invasivo en cuerpos de agua (Ma et al., 2010; Nigam, 2002). Para la transformación en etanol de estas malezas se requiere la adopción de métodos costo efectivos de pretratamiento, detoxificación, sacarificación y fermentación para sus tecnologías de producción (Aswathy et al., 2010). Es importante agregar, que la etapa de pretratamiento de lignocelulósicos ha sido reconocida como una barrera tecnológica para el desarrollo rentable de bioprocesos a partir de materiales lignocelulósicos (Cheng et al., 2008), de modo que existe la necesidad de conocer el potencial de diferentes pretratamientos con el Buchón de Agua, con la finalidad de elegir el más conveniente.

Mishima et al. (2006) investigaron la hidrólisis de biomasa de Buchón de Agua pretratado mediante 20 pretratamientos químicos. En su estudio el pretratamiento de las hojas de Buchón de Agua con agua destilada a $100^{\circ} \mathrm{C}$ por $2 \mathrm{~h}$ permitió obtener $8,2 \mathrm{mg}$ de azúcares reductores/ $100 \mathrm{mg}$ biomasa pretratada. El pretratamiento de la misma biomasa con $\mathrm{NaOH}$ al 5 y $10 \%$ a temperatura ambiente por $2 \mathrm{~h}$ permitió obtener 8,8 y $9,3 \mathrm{mg}$ de azúcares reductores/ $100 \mathrm{mg}$ biomasa pretratada, respectivamente. El mismo pretratamiento con $\mathrm{NaOH}$ al $1 \%$ por $100^{\circ} \mathrm{C}$ y $2 \mathrm{~h}$ dio como resultado $8,9 \mathrm{mg}$ de azúcares reductores/ $100 \mathrm{mg}$ biomasa pretratada. El mejor resultado (11 mg de azúcares reductores/ $100 \mathrm{mg}$ biomasa pretratada) fue el obtenido con pretratamiento denominado Alcalino/Oxidativo a temperatura ambiente por $24 \mathrm{~h}$, que se realizó en dos etapas: $12 \mathrm{~h}$ de pretratamiento con $\mathrm{NaOH} 1 \%$ (peso/volumen) y $12 \mathrm{~h}$ adicionales con $\mathrm{H}_{2} \mathrm{O}_{2} 31 \%$ (peso/volumen) (Mishima et al., 2006).

Otro trabajo sobresaliente es el de Ganguly et al., (2013). En este los investigadores pretrataron biomasa seca de Buchón de Agua con $\mathrm{NaOH} 5 \%$ por 10 min a $150^{\circ} \mathrm{C}$. El mayor rendimiento de azúcares reductores fue de $465 \mathrm{mg} / \mathrm{g}$ de biomasa al llevar a cabo la hidrólisis con celulasas $(103,75 \mathrm{U} / \mathrm{g})$ en shaker a $50^{\circ} \mathrm{C}$ y 75 rpm por 12h. La fermentación con Saccharomyces cerevisiae arrojó una producción de etanol fue de 3,07 $\mathrm{g} / \mathrm{ml}$ (Ganguly et al., 2013). El efecto del ácido diluido también ha sido estudiado previamente. Guragain et al., (2011) pretrataron $15 \mathrm{~g}$ de biomasa de Buchón de Agua con $300 \mathrm{ml} \mathrm{de} \mathrm{H}_{2} \mathrm{SO}_{4} 1 \%$ a $140^{\circ} \mathrm{C}$ por 40 min. En la etapa de hidrólisis el material pretratado dio un rendimiento de azúcares reductores de $714 \mathrm{mg} / \mathrm{g}$ muestra y la etapa de fermentación un rendimiento de etanol de $445 \mathrm{mg}$ glucosa/g muestra (Guragain et al., 2011). Como puede observarse, los reportes a la fecha no muestran una comparación detallada de los pretratamientos, a la vez que no registran optimización de condiciones de operación.

Este trabajo tiene como objetivo caracterizar la composición del Buchón de Agua en cenizas, extractivos, carbohidratos estructurales y lignina, observar el efecto de diferentes pretratamientos sobre la biomasa de Buchón de Agua y determinar las mejores condiciones de operación en los pretratamientos que arrojen los mejores resultados. Igualmente, la aplicación de pretratamientos y evaluación de la capacidad hidrolítica y fermentativa son objeto de este estudio, permitiendo la selección de un proceso más adecuado para la preparación del material para las etapas posteriores.

\section{METODOLOGÍA}

El Buchón de Agua utilizado es proveniente de la represa Guatapé de Empresas Públicas de Medellín ubicada en el oriente del departamento de Antioquia a una altura de $1887,5 \mathrm{msnm}$, la cual cuenta con una potencia instalada de $560 \mathrm{MW}$ y una capacidad de $107^{\prime} 021.000 \mathrm{~m}^{3}$ (Aguirre et al., 2007). Este material fue secado al aire inicialmente para facilitar su manipulación, posteriormente fue sometido a molienda hasta una granulometría inferior a $3 \mathrm{~mm}$ y finalmente, secado hasta una humedad inferior al $10 \%$ (wt.).

\section{Determinación de celulosa, hemicelulosa y lignina en biomasa}

La biomasa pretratada y sin pretratar de Buchón de Agua se sometió a una caracterización fisicoquímica siguiendo los protocolos del National Renewable Energy Laboratory, determinándose parámetros como cenizas y humedad. Los contenidos de celulosa, hemicelulosa y lignina en los materiales pretratados y sin pretratar se determinaron por espectroscopia UV-vis, posterior a la hidrólisis ácida con $72 \%$ de $\mathrm{H}_{2} \mathrm{SO}_{4}$. Los azúcares reductores totales (ATR) fueron determinados por el método de ácido 3,5-dinitrosalicílico (DNS) de Miller (1959) y la glucosa se determinó usando el kit de oxidasa/peroxidasa de BioSystems $\AA^{\circledR}$. Los contenidos de etanol en los puntos finales de las fermentaciones fueron determinados por cromatografía de gases utilizando un cromatógrafo Agilent 7890 con detector FID empleando una columna HP - INNOWax acoplado a microextracción en fase sólida (SPME) con una fibra de poliacrilato 85 - LM. 


\section{Determinación de cenizas y extractivos (NREL, 2008)}

Para la determinación de cenizas, se calcinó el material lignocelulósico a una temperatura de $575{ }^{\circ} \mathrm{C}$ (Sluiter et al., 2005). La determinación de extractivos, se realizó con un equipo de extracción soxhlet; la temperatura de calentamiento del solvente (etanol) fue de $115^{\circ} \mathrm{C}$. En ambos casos se determinó por gravimetría respecto a la biomasa seca los contenidos.

\section{Evaluación Preliminar de técnicas de pretratamiento}

Las condiciones empleadas corresponden a información reportada en la literatura bajo las que se obtienen altos rendimientos de azúcares fermentables. Cada pretratamiento se realizó en sistemas de agitación con control de la temperatura a las siguientes condiciones: Deslignificación alcalina con $\mathrm{NaOH}$ : Relación Biomasa/Solución 1:15 (wt.), $\mathrm{NaOH} 1 \%, 120^{\circ} \mathrm{C}$ y 30 minutos; Hidrolisis con ácido diluido: Relación Biomasa/Solución 1:10 (w:v), $\mathrm{H}_{2} \mathrm{SO}_{4} 2 \%, 90^{\circ} \mathrm{C}$ y 90 minutos; Remojo con solución de Amoniaco: Relación Biomasa/Solución 1:8 (w:v), $\mathrm{NH}_{4} \mathrm{OH} 15 \%, 60^{\circ} \mathrm{C}$ y 6 horas; Peróxido alcalino: Relación Biomasa/Solución 1:20 (wt.), $\mathrm{H}_{2} \mathrm{O}_{2} 2 \%, \mathrm{pH} 11,5 \mathrm{con} \mathrm{NaOH} 10 \mathrm{M}, 35^{\circ} \mathrm{C}$ y 3 horas. Todas las soluciones fueron preparadas con agua destilada. Finalmente se separaron por filtración las fracciones sólidas y líquidas, las fracciones sólidas se lavaron con agua de grifo en proporción Fracción sólida/Agua 1:6, efectuando varios lavados hasta que presentaron un $\mathrm{pH}$ de 7 y se secaron.

\section{Evaluación de la capacidad hidrolítica y fermentativa de los materiales}

Se esterilizó el material pretratado a $121^{\circ} \mathrm{C}$ por 15 minutos y se usó la enzima comercial Accellerase 1500 (Genencor) en una proporción de $30 \mathrm{FPU} / \mathrm{g}$ de biomasa en una solución buffer de citrato de sodio (50 mM, $\mathrm{pH}$ 4,8). La mezcla fue incubada a $50^{\circ} \mathrm{C}$ y $180 \mathrm{rpm}$ en agitación orbital. El sistema fue monitoreado durante 72 horas, realizándose seguimiento al contenido de azúcares reductores totales (ATR). Por otra parte, para determinar la capacidad fermentativa se aplicó un método de Hidrólisis y Fermentación Simultaneas (SSF), adicionando la levadura comercial Saccharomyces cerevisiae Ethanol Red® en una proporción de $2 \mathrm{~g} / \mathrm{L}$ de levadura seca después de $12 \mathrm{~h}$ de iniciada la hidrólisis, disminuyendo la temperatura a $37^{\circ} \mathrm{C}$ y la agitación a $150 \mathrm{rpm}$ en anaerobiosis. El seguimiento se hizo monitoreando la pérdida de peso y el punto final de concentración de etanol en el medio se determinó mediante cromatografía de gases acoplada a un sistema de micro-extracción en fase sólida (SPME).

\section{Evaluación del Pretratamiento Ácido Diluido}

Se estudiaron las variables tiempo, temperatura, concentración de agente químico y relación sólido/líquido, para maximizar el rendimiento de azúcares reductores y etanol. La Tabla 1 muestra los rangos de las variables. Vale aclarar que este pretratamiento fue escogido una vez analizados los resultados iniciales. Se aplicó un diseño Factorial $2^{4}$ de 1 bloque y 2 respuestas en 18 corridas, 2 puntos centrales por bloque; y 7 Grados de libertad para el error. Los rangos fueron escogidos de acuerdo a reportes de la literatura (Isarankura-Na-Ayudhya et al., 2007; Kumar et al., 2009; Masami et al., 2008).

Tabla 1. Variables de estudio del diseño experimental pretratamiento Ácido Diluido

\begin{tabular}{|l|c|c|c|l|c|}
\hline Factores & Bajo & Alto & Unidades & Respuestas & Unidades \\
\cline { 1 - 4 } Temperatura & 80 & 121 & ${ }^{\circ} \mathrm{C}$ & Rendimiento de Etanol & $\mathrm{g} / \mathrm{L}$ \\
\hline Tiempo & 30 & 90 & Minutos & Rendimiento de ATR & $\mathrm{g} / \mathrm{L}$ \\
\cline { 1 - 4 } Relación, s/l & 0.5 & 2 & $(\% \mathrm{w} / \mathrm{v})$ & & \\
\cline { 1 - 3 } $\mathrm{H}_{2} \mathrm{SO}_{4} \%$ & 5 & 10 & $(\% \mathrm{w} / \mathrm{w})$ & &
\end{tabular}

La Tabla 2 muestra las condiciones de los diferentes experimentos llevados a cabo para la optimización del pretratamiento Ácido Diluido.

Tabla 2. Diseño Factorial para la optimización del pretratamiento Ácido Diluido

\begin{tabular}{|c|c|c|c|c|}
\hline Experimento & Temperatura $\left({ }^{\circ} \mathrm{C}\right)$ & Tiempo $(\mathrm{min})$ & $\begin{array}{c}\text { Concentración } \mathrm{H}_{2} \mathrm{SO}_{4}(\% \\
\text { peso/peso) }\end{array}$ & $\begin{array}{c}\text { Relación s/l } \\
\text { (\% peso/volumen) }\end{array}$ \\
\hline 1 & 80 & 30 & 0,5 & 5 \\
\hline 2 & 100,5 & 60 & 1,25 & 5,5 \\
\hline 3 & 80 & 90 & 2 & 5 \\
\hline 4 & 121 & 30 & 2 & 5 \\
\hline 5 & 80 & 30 & 1,25 & 7,5 \\
\hline 6 & 100,5 & 60 & 2 & 5 \\
\hline
\end{tabular}


Tabla 2 (continuación)

\begin{tabular}{|c|c|c|c|c|}
\hline 7 & 80 & 30 & 0,5 & 10 \\
\hline 8 & 121 & 30 & 0,5 & 10 \\
\hline 9 & 121 & 90 & 0,5 & 5 \\
\hline 10 & 121 & 30 & 0,5 & 5 \\
\hline 11 & 80 & 90 & 2 & 10 \\
\hline 12 & 80 & 30 & 2 & 10 \\
\hline 13 & 121 & 30 & 2 & 10 \\
\hline 14 & 80 & 90 & 0,5 & 10 \\
\hline 15 & 121 & 90 & 2 & 5 \\
\hline 16 & 121 & 90 & 0,5 & 10 \\
\hline 17 & 80 & 90 & 2 & 10 \\
\hline 18 & 121 & 90 & & \\
\hline
\end{tabular}

\section{Evaluación del Pretratamiento Deslignificación Alcalina}

Para optimizar las condiciones del pretratamiento deslignificacion alcalina y maximizar la producción de azúcares y etanol se propuso un diseño de superficie de respuesta compuesto reducido Draper - Lin, mediante el cual estudiaron los efectos de 4 factores en 18 corridas. La muestra los datos del diseño.

Tabla 3. Variables de estudio del diseño experimental pretratamiento Deslignificación Alcalina

\begin{tabular}{|l|c|c|c|l|c|}
\hline Factores & Bajo & Alto & Unidades & Respuestas & Unidades \\
\cline { 1 - 4 } Temperatura & 80 & 120 & ${ }^{\circ} \mathrm{C}$ & Rendimiento de Etanol & $\mathrm{g} / \mathrm{L}$ \\
\hline Tiempo & 60 & 180 & Minutos & Rendimiento de ATR & $\mathrm{g} / \mathrm{L}$ \\
\cline { 1 - 4 } Relación s/l & $1 / 15$ & $1 / 20$ & $(\% \mathrm{w} / \mathrm{v})$ & & \\
\cline { 1 - 4 } $\mathrm{NaOH} \%$ & 1 & 2 & $(\% \mathrm{w} / \mathrm{w})$ & &
\end{tabular}

La Tabla 4 muestra las condiciones de los diferentes experimentos llevados a cabo para la optimización del pretratamiento Deslignificación Alcalina.

Tabla 4. Diseño experimental del tipo Draper-Lin para la optimización del pretratamiento alcalino

\begin{tabular}{|c|c|c|c|c|}
\hline Experimento & Temperatura $\left({ }^{\circ} \mathrm{C}\right)$ & Tiempo (min) & $\begin{array}{c}\text { Concentración } \mathrm{NaOH} \\
\text { (\% peso/peso) }\end{array}$ & $\begin{array}{c}\text { Relación s/l } \\
\text { (\% peso/volumen) }\end{array}$ \\
\hline 1 & 100 & 120 & 2,3 & 17,5 \\
\hline 2 & 80 & 60 & 2 & 15 \\
\hline 3 & 100 & 120 & 0,66 & 17,5 \\
\hline 4 & 66,4 & 120 & 1,5 & 17,5 \\
\hline 5 & 120 & 60 & 2 & 20 \\
\hline 6 & 120 & 60 & 1 & 20 \\
\hline 7 & 80 & 180 & 1 & 20 \\
\hline 8 & 80 & 60 & 1 & 15 \\
\hline 9 & 80 & 180 & 2 & 20 \\
\hline 10 & 133,6 & 120 & 1,5 & 17,5 \\
\hline 11 & 100 & 19,2 & 1,5 & 17,5 \\
\hline 12 & 100 & 222 & 1,5 & 17,5 \\
\hline 13 & 100 & 120 & 1,5 & 17,5 \\
\hline 14 & 100 & 120 & 1,5 & 17,5 \\
\hline 15 & 100 & 120 & 1,5 & 13,3 \\
\hline 16 & 120 & 180 & 1 & 15 \\
\hline 17 & 120 & 180 & 2 & 15 \\
\hline 18 & 100 & 120 & 1,5 & 21,7 \\
\hline
\end{tabular}




\section{RESULTADOS Y DISCUSIÓN}

A continuación, se presentan los resultados obtenidos en cuanto a la composición del Buchón de Agua, la selección de pretratamientos y la optimización de los pretratamientos con Ácido Diluido y Deslignificación Alcalina.

\section{Composición Buchón de Agua}

La composición del Buchón de Agua suministrado por Empresas Públicas de Medellín se encuentra dentro de los rangos encontrados en la literatura. El contenido de celulosa de 19,61\% y el de hemicelulosa con un $35,15 \%$ se acercan al reportado en la literatura (Bhattacharya y Kumar, 2002; Sornvoraweat y Kongkiattikajorn, 2010). La composición en carbohidratos de $54,76 \%$ indica que más de la mitad de la composición en peso seco del material es en azúcares. La Tabla 5 presenta los cambios en la composición de la biomasa por acción de los pretratamientos en la etapa se selección preliminar.

Tabla 5. Composición de la fracción sólida de Buchón de Agua antes y después de la selección preliminar de pretratamientos (\%peso, base seca)

\begin{tabular}{|l|c|c|c|c|c|c|}
\hline Material/Pretratamiento & Celulosa & Hemicelulosa & Lignina & Extractivos & Cenizas & Otros \\
\hline No pretratado & 19,61 & 35,15 & 28,85 & 1,05 & 9,44 & 5,90 \\
\hline $\mathrm{NaOH}$ & 25,73 & 39,45 & 23,21 & 1,18 & 4,82 & 5,61 \\
\hline Ácido diluido & 22,09 & 37,87 & 24,95 & 2,04 & 7,14 & 5,92 \\
\hline Amoniaco Acuoso & 22,42 & 37,53 & 21,52 & 7,53 & 3,40 & 7,60 \\
\hline Peróxido Alcalino & 23,05 & 37,30 & 15,00 & 1,01 & 4,59 & 10,46 \\
\hline
\end{tabular}

La fracción denominada Otros puede incluir compuestos menores como aceites, proteínas, minerales, etc. (Ganguly y Saha, 2013); a composición de las cenizas en el material incluyen $\mathrm{K}_{2} \mathrm{O}, \mathrm{Na}_{2} \mathrm{O}, \mathrm{CaO}$ y $\mathrm{Cl}$ (Ahn et al., 2012). Aplicando pretratamiento con ácido diluido se consiguió una recuperación de sólidos de las más altas $(64,66 \%)$, con un $55,92 \%$ de lignina y un $30,34 \%$ de Hemicelulosa removidas del material, que en comparación con $25 \%$ y $31,2 \%$ de lignina y hemicelulosa de la literatura (Singh y Bishnoi, 2013), resultado favorable, pues se consiguió una conversión de azúcares a etanol de 59,46\% (Figura 2), superior a la de los demás pretratamientos.

Por otra parte, es sabido que los pretratamientos alcalinos son básicamente procesos de deslignificación en donde una cantidad significativa de hemicelulosa también es solubilizada (Ganguly y Saha, 2013). En la tabla 2 se evidencia un aumento en la composición de carbohidratos luego del pretratamiento deslignificación alcalina. Para tratamientos similares reportados en la literatura, a concentraciones superiores de álcali (2\%), se recuperaron cantidades inferiores de celulosa (68\%) y hemicelulosa (37\%), pero con remociones de lignina superiores (56\%) (Singh y Bishnoi, 2013). El tratamiento de remojo con amoniaco presentó la más baja recuperación de sólidos y remoción de lignina (tabla 2). Esta última fue relativamente baja en comparación a un 59,3\% reportado en pasto varilla (switchgrass), que contiene cantidades similares de lignina (Wyman et al., 2011). El Pretratamiento del peróxido alcalino, pese al efecto de deslignificación del material y las altas recuperaciones de carbohidratos estructurales, fue el que menos afectó la estructura del material, lo cual se evidencia en las bajas pérdidas de sólidos y el trabajo casi nulo de la enzima y la levadura en la fase de hidrolisis y fermentación.

\section{Curvas Cinéticas de producción de azúcares reductores y etanol (SSF).}

Se realizó un seguimiento a la cinética de producción de azúcares reductores y etanol para cada una de las fracciones sólidas provenientes de los cuatro pretratamientos. La

Fig. 1 muestra los datos de las cinéticas de producción de azúcares. Los materiales que exhibieron las mejores producciones de azúcares reductores fueron los pretratados con deslignificacion alcalina y remojo con amoníaco con 155,51 mg/g (27,2 g/L) y 109,83 mg/g (20,4 g/L), alcanzando la fase estacionaria a las 8 horas. Los resultados de hidrólisis, en su orden, fueron el ácido diluido y el peróxido alcalino con 95,91 mg/g $(14,1 \mathrm{~g} / \mathrm{L})$ y $34,97 \mathrm{mg} / \mathrm{g}(4,2 \mathrm{~g} / \mathrm{L})$. La mayoría superan por mucho los reportados en la literatura de $13,5 \mathrm{~g} / \mathrm{L}$ para pretratamiento alcalino (Singh y Bishnoi, 2013). En el caso de la producción de etanol mediante el sistema de Sacarificación y Fermentación simultaneas SSF, se obtuvo resultados muy promisorios (

Fig. 2). A las 9 horas de fermentación, con los pretratamientos deslignificacion alcalina, ácido diluido, Remojo con amoníaco y el Peróxido alcalino se observaron rendimientos de 71,34 mg/g (12,51 g/L), 57,03 $\mathrm{mg} / \mathrm{g}(8,41 \mathrm{~g} / \mathrm{L}), 53,59 \mathrm{mg} / \mathrm{g}(9,96 \mathrm{~g} / \mathrm{L})$ y $11,21 \mathrm{mg} / \mathrm{g}(1,35 \mathrm{~g} / \mathrm{L})$ de etanol. Claramente estos resultados 
superan reportes de literatura de 9,8 $\pm 2.2 \mathrm{~g} / \mathrm{L}$ (Singh y Bishnoi, 2013). Debe considerarse que toda la información correspondiente los pretratamientos se desarrolló por duplicado, con errores en los resultados menores al $5 \%$.

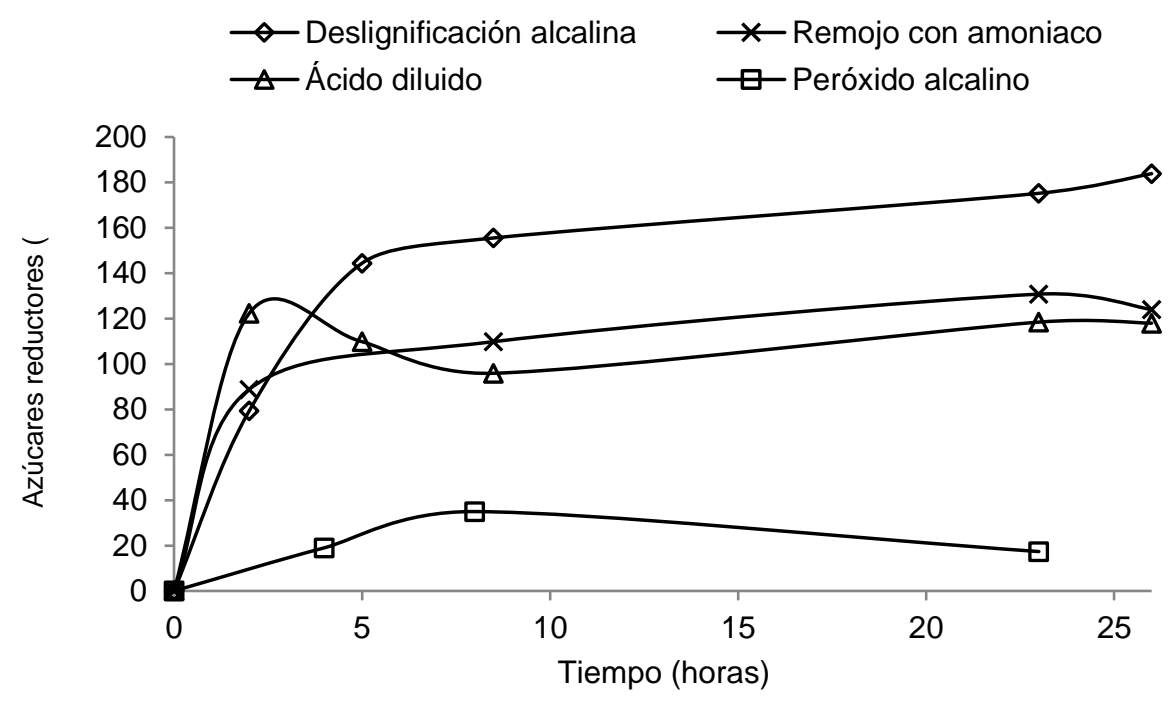

Fig. 1. Curvas cinéticas de la producción de azúcares reductores de los pretratamientos. Los Azúcares reductores están en (mg ATR/g biomasa sin pretratar seca)

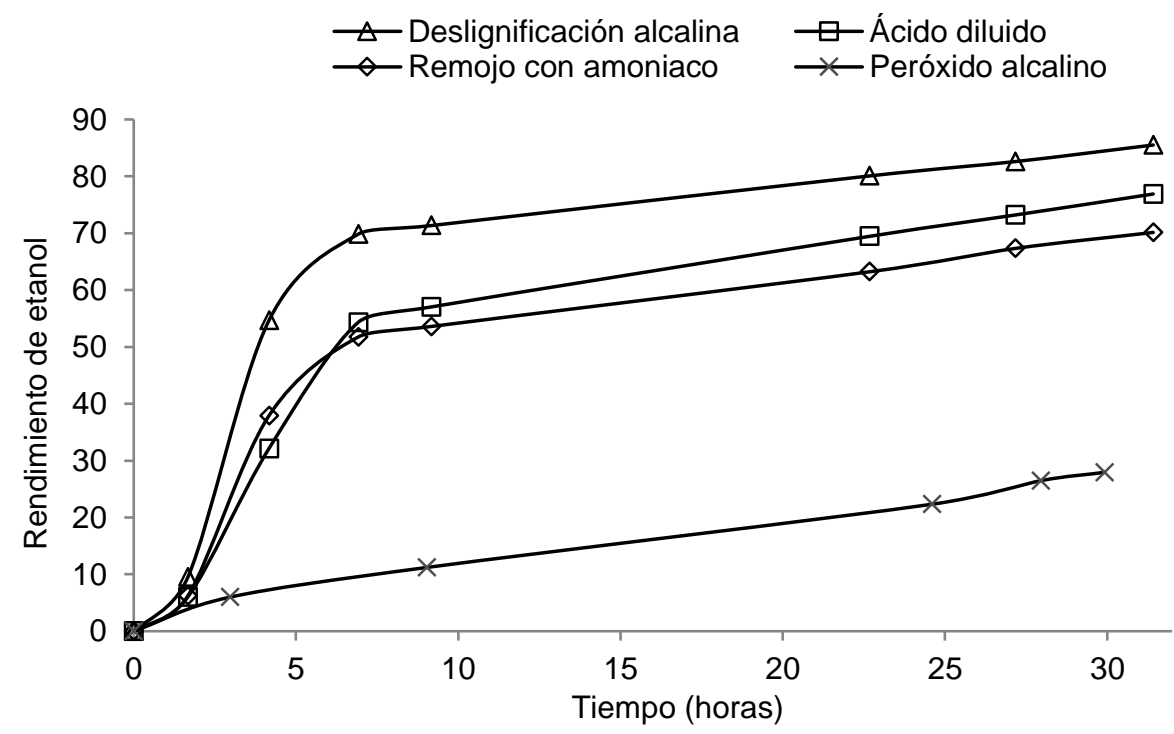

Fig. 2. Curvas de fermentación de acuerdo a los pretratamientos aplicados a Buchón de Agua. Rendimiento de etanol está en (mg EtOH/g biomasa sin pretratar seca)

\section{Composición de Fracción Sólida de Buchón de Agua pretratado con ácido diluido}

Se hizo un seguimiento a la producción de azúcares y de etanol, de igual modo se estudió la cantidad de sólidos recuperados y de carbohidratos estructurales, en tanto los primeros incrementan la posibilidad de producción de etanol respecto a la cantidad de material alimentado al tiempo que, la recuperación de celulosa y solubilización de la hemicelulosa sugiere un aumento de la porosidad del material y digestibilidad enzimática a merced de la disminución de enlaces entre la lignina y la celulosa (Mateus et al., 2012).

La información consignada en la Tabla 6 permite observar qué tanto surte efecto el pretratamiento en el material, notándose que efectivamente el pretratamiento No. 18 que se operó a $121^{\circ} \mathrm{C}$ durante 90 minutos con un $2 \%$ de agente químico y $10 \%$ de concentración de sólidos permitiría recuperar una gran cantidad de la celulosa en la fracción sólida $(98,0 \%)$, lo cual convierte a este pretratamiento en el más eficiente en la extracción de este componente orgánico de la biomasa de Buchón de Agua entre todos los estudiados.

Tabla 6. Composición de Fracción Sólida de Buchón de Agua pretratado con $\mathrm{H}_{2} \mathrm{SO}_{4}$ diluido 


\begin{tabular}{|c|c|c|c|c|c|c|c|}
\hline Exp. & $\begin{array}{c}\text { Celulosa } \\
(\%)\end{array}$ & $\begin{array}{c}\text { H-celulosa } \\
(\%)\end{array}$ & $\begin{array}{c}\text { Lignina } \\
(\%)\end{array}$ & $\begin{array}{c}\text { Recuperación } \\
\text { sólido (\%) }\end{array}$ & $\begin{array}{c}\text { Recuperación } \\
\text { Celulosa (\%) }\end{array}$ & $\begin{array}{c}\text { Recuperación } \\
\text { H-celulosa (\%) }\end{array}$ & $\begin{array}{c}\text { Lignina } \\
\text { Removida (\%) }\end{array}$ \\
\hline 1 & 22,8 & 10,2 & 24,3 & 69,6 & 80,8 & 20,3 & 58,5 \\
\hline 2 & 27,6 & 1,0 & 31,5 & 65,8 & 92,6 & 1,9 & 71,9 \\
\hline 3 & 29,3 & 6,6 & 24,3 & 64,0 & 95,6 & 12,1 & 53,9 \\
\hline 4 & 29,1 & 4,6 & 33,1 & 60,5 & 89,8 & 7,9 & 69,3 \\
\hline 5 & 25,1 & 1,9 & 30,1 & 61,3 & 78,5 & 3,3 & 63,9 \\
\hline 6 & 18,8 & 25,2 & 24,4 & 76,9 & 73,8 & 55,1 & 65,0 \\
\hline 7 & 25,4 & 14,8 & 22,4 & 62,5 & 81,0 & 26,3 & 48,6 \\
\hline 8 & 24,6 & 0,2 & 24,5 & 77,5 & 97,4 & 0,4 & 65,7 \\
\hline 9 & 28,5 & 9,7 & 36,6 & 47,4 & 68,8 & 13,1 & 60,2 \\
\hline 10 & 23,3 & 2,6 & 25,8 & 62,7 & 74,3 & 4,6 & 56,1 \\
\hline 11 & 24,1 & 24,3 & 34,2 & 80,0 & 98,5 & 55,2 & 94,8 \\
\hline 12 & 18,7 & 28,2 & 19,9 & 83,8 & 80,0 & 67,2 & 57,8 \\
\hline 13 & 21,1 & 6,5 & 25,2 & 69,4 & 74,6 & 12,9 & 60,7 \\
\hline 14 & 24,6 & 4,4 & 23,8 & 77,0 & 96,7 & 9,6 & 63,6 \\
\hline 15 & 28,1 & 24,9 & 29,1 & 46,2 & 66,3 & 32,7 & 46,6 \\
\hline 16 & 31,2 & 0,8 & 32,5 & 62,0 & 98,7 & 1,4 & 69,8 \\
\hline 17 & 22,5 & 6,7 & 20,7 & 80,0 & 91,8 & 15,3 & 57,5 \\
\hline 18 & 29,1 & 1,9 & 39,3 & 66,0 & 98,0 & 3,6 & 90,0 \\
\hline
\end{tabular}

\section{Optimización de Pretratamiento Hidrólisis con ácido diluido}

Se presentan los resultados obtenidos en términos de producción de azúcares reductores y etanol respecto a las cantidades de biomasa alimentada, lo cual permite visualizar los rendimientos reales, a diferencia de las variables de respuesta elegidas que se expresan en gramos por litro, como concentraciones en caldo. Los resultados se muestran en la Fig. 3.

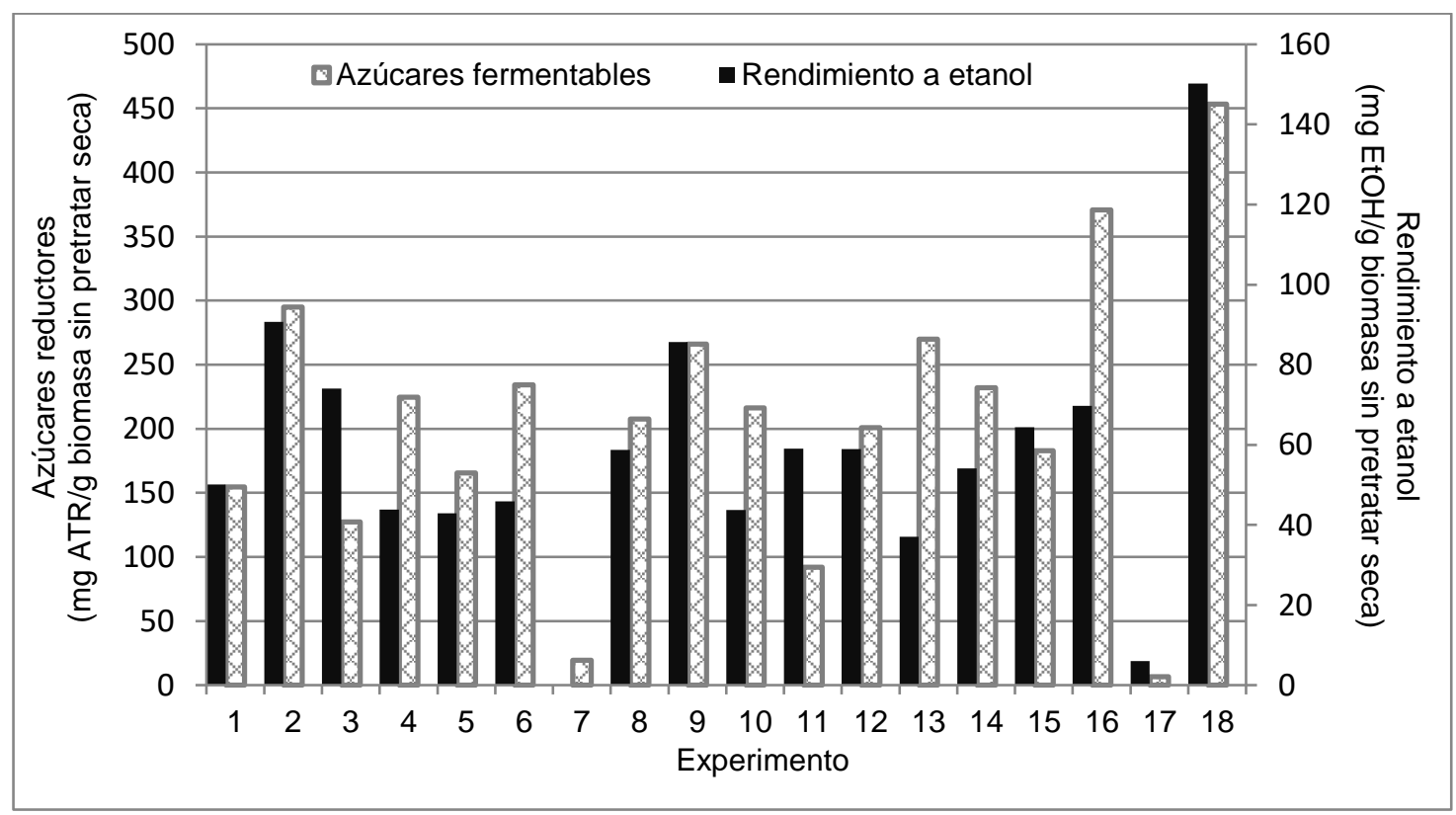

Fig. 3. Rendimiento de azúcares reductores totales (mg ATR/g biomasa sin pretratar seca) y rendimiento de etanol (mg $\mathrm{EtOH} / \mathrm{g}$ biomasa sin pretratar seca) de cada uno de los experimentos del pretratamiento ácido diluido

Con las condiciones de pretratamiento implementadas con ácido diluido, se obtuvo rendimientos de azúcares reductores muy variables, que oscilaron entre $6,7 \mathrm{mg}$ de ATR/g biomasa seca sin pretratar y 453,31 mg de ATR/g de biomasa. La cantidad de azúcares reductores obtenida se acerca o supera valores alcanzados con pretratamientos ácidos reportados en la literatura de 293,3 mg/g (Reales-Alfaro et al., 2013), 
$483 \mathrm{mg} / \mathrm{g}$ (Xia et al., 2013), $375 \mathrm{mg} / \mathrm{g}$ (Abraham y Kurup, 1996). Los pretratamientos 16 y 18 del diseño fueron los más eficientes, teniendo en común las altas recuperaciones de celulosa en la fracción sólida. Igualmente, tienen en común que se encuentran en el nivel superior de las temperaturas tiempos y concentraciones de ácido exploradas por el diseño $\left(121^{\circ} \mathrm{C}, 90\right.$ minutos y $2 \%$ respectivamente). La Fig. 3 muestra también los resultados de fermentación. Los rendimientos de etanol corresponden a 28 horas de fermentación. El más alto, de 150,19 mg EtOH/g es bastante alentador en comparación con rendimientos reportados de $4.25 \mathrm{~g} / \mathrm{L}$ en 84 horas de fermentación (Aswathy et al., 2010), $146 \mathrm{mg} / \mathrm{g}$ (Ma et al., 2010) por 72 horas, 116 mg/g (Abraham y Kurup, 1996).

La Tabla 7 muestra los resultados del diseño experimental llevado a cabo para hallar las mejores condiciones de operación para el pretratamiento hidrólisis ácida. Los resultados finales permiten determinar que es posible producir $150,19 \mathrm{mg}$ de etanol/g biomasa seca sin pretratar a partir la fracción sólida (correspondiente a 190,35 litros etanol/ton biomasa seca alimentada) obtenida del pretratamiento con ácido del Buchón de Agua (fermentación SSF) a unas condiciones de $121^{\circ} \mathrm{C}$ de temperatura, 90 minutos de tiempo de residencia, $2 \%$ de concentración de $\mathrm{H}_{2} \mathrm{SO}_{4}$ y $10 \%$ de concentración de sólidos. Esta información permitió concluir que efectivamente dichas condiciones se encuentran en el límite superior de los rangos ensayados. El óptimo sugiere la posibilidad de explorar condiciones más extremas que las ensayadas en el actual diseño, en miras de conseguir mejores rendimientos de azúcares y etanol.

Tabla 7. Resultados del diseño experimental

\begin{tabular}{|c|c|c|c|c|c|c|}
\hline Factor & Bajo & Alto & Óptimo & Respuesta & Óptimo & Validado \\
\hline Temperatura $\left({ }^{\circ} \mathrm{C}\right)$ & 80,0 & 121,0 & 121,00 & Etanol (g/L) & 15,68 & 18,00 \\
\hline Tiempo (min) & 30,0 & 90,0 & 90,00 & ATR $(\mathrm{g} / \mathrm{L})$ & 51,00 & 51,10 \\
\hline$\left[\mathrm{H}_{2} \mathrm{SO}_{4}\right]$ (\%peso) & 0,5 & 2,0 & 1,91 & & & \\
\hline Relación sólido líquido (g/g) & 5,0 & 10,0 & 10,00 & & & \\
\hline
\end{tabular}

\section{Composición de Fracción Sólida de Buchón de Agua pretratado con deslignificación alcalina}

Al igual que en el pretratamiento anterior, en la Tabla 6 se presenta la composición de los materiales pretratados incluyéndose la recuperación de los sólidos y carbohidratos estructurales, además de la remoción de lignina que en este caso cobra importancia porque este tipo de técnicas se caracterizan precisamente por tener dicho efecto (Ganguly et al., 2012).

Tabla 8. Composición de los experimentos del pretratamiento deslignificación con $\mathrm{NaOH}$ en Buchón de Agua

\begin{tabular}{|c|c|c|c|c|c|c|c|}
\hline Exp. & $\begin{array}{c}\text { Celulosa } \\
(\%)\end{array}$ & $\begin{array}{c}\text { H-celulosa } \\
(\%)\end{array}$ & $\begin{array}{c}\text { Lignina } \\
(\%)\end{array}$ & $\begin{array}{c}\text { Recuperación } \\
\text { sólido (\%) }\end{array}$ & $\begin{array}{c}\text { Recuperación } \\
\text { Celulosa (\%) }\end{array}$ & $\begin{array}{c}\text { Recuperación } \\
\text { H-celulosa (\%) }\end{array}$ & $\begin{array}{c}\text { Lignina } \\
\text { removida (\%) }\end{array}$ \\
\hline 1 & 16,0 & 14,7 & 28,6 & 65,4 & 53,4 & 27,4 & 35,2 \\
\hline 2 & 12,2 & 19,4 & 31,2 & 77,7 & 48,2 & 42,9 & 15,9 \\
\hline 3 & 11,5 & 24,7 & 30,8 & 63,7 & 37,3 & 44,7 & 31,9 \\
\hline 4 & 12,1 & 25,5 & 31,8 & 86,8 & 53,7 & 62,9 & 4,4 \\
\hline 5 & 12,0 & 15,3 & 38,9 & 59,4 & 36,2 & 25,9 & 20,0 \\
\hline 6 & 9,7 & 18,3 & 38,4 & 57,5 & 28,3 & 29,9 & 23,4 \\
\hline 7 & 12,9 & 23,0 & 37,3 & 70,3 & 46,1 & 46,0 & 9,1 \\
\hline 8 & 20,6 & 24,1 & 25,6 & 88,8 & 93,3 & 60,8 & 21,2 \\
\hline 9 & 12,9 & 18,0 & 40,0 & 64,5 & 42,3 & 33,0 & 10,6 \\
\hline 10 & 20,1 & 47,4 & 11,7 & 67,4 & 69,1 & 91,0 & 72,7 \\
\hline 11 & 12,5 & 9,0 & 10,9 & 61,8 & 39,4 & 15,9 & 76,7 \\
\hline 12 & 13,3 & 16,6 & 15,0 & 72,6 & 49,2 & 34,3 & 62,2 \\
\hline 13 & 11,1 & 15,2 & 14,5 & 77,0 & 43,6 & 33,3 & 61,4 \\
\hline 14 & 13,7 & 17,8 & 38,9 & 73,1 & 52,6 & 38,1 & 1,5 \\
\hline 15 & 9,1 & 22,5 & 29,6 & 75,0 & 35,0 & 48,0 & 22,9 \\
\hline 16 & 15,3 & 14,6 & 25,1 & 58,3 & 45,6 & 24,2 & 49,4 \\
\hline 17 & 13,8 & 14,1 & 29,3 & 69,6 & 48,9 & 27,9 & 29,3 \\
\hline 18 & 10,0 & 15,3 & 16,0 & 83,7 & 42,7 & 36,4 & 53,7 \\
\hline
\end{tabular}

Los pretratamientos 8 y 10 presentan porcentajes considerables de recuperación de celulosa $(93,34)$ y hemicelulosa $(90,95)$, siendo este último también muy eficiente en la remoción de lignina, esto implica que 
las fracciones sólidas provenientes de estos tratamientos prometen resultados muy eficientes en cuanto a la liberación de azúcares y subsecuente producción de etanol.

\section{Optimización de Pretratamiento Deslignificación Alcalina}

Con las condiciones de pretratamiento, se obtuvieron rendimientos de azúcares reductores que oscilaron entre $214,63 \mathrm{mg}$ de ATR/g de biomasa y $347,48 \mathrm{mg}$ de ATR/g de biomasa después de 30 horas de reacción, tal como se muestra en la

Fig. 4. Los rendimientos y concentraciones de azúcares reductores están acordes y en muchos casos superan a los de la literatura de $483 \mathrm{mg} / \mathrm{g}$ (Xia et al., 2013), $375 \mathrm{mg} / \mathrm{g}$ (Abraham, 1996). Los rendimientos de etanol oscilan entre $46,93 \mathrm{mg} \mathrm{EtOH} / \mathrm{g}$ y $155,69 \mathrm{mg} \mathrm{EtOH} / \mathrm{g}$ de biomasa sin pretratar seca luego de 28 horas de fermentación. La

Fig. 4 muestra también que los experimentos 4,5 y 8 exhiben los mejores rendimientos con 155,69 mg $\mathrm{EtOH} / \mathrm{g}(18,53 \mathrm{~g} / \mathrm{L}), 147,06 \mathrm{mg} \mathrm{EtOH} / \mathrm{g}(22,25 \mathrm{~g} / \mathrm{L})$ y $152,74 \mathrm{mg} \mathrm{EtOH} / \mathrm{g}(17,11 \mathrm{~g} / \mathrm{L})$. Esta información muestra que se alcanzaron concentraciones superiores a las reportadas en la literatura, como $14,4 \mathrm{~g} / \mathrm{L}$ con S. cereviciae 16,9 g/L con una cepa de Escherichia coli KO11 (Mishima et al., 2008), 4,5 g/L (110mg/g sustrato) con el método SSF (Mukhopadhyay, 2010) y 7,37g/L con S. cerevisiae (Ahn et al., 2011).

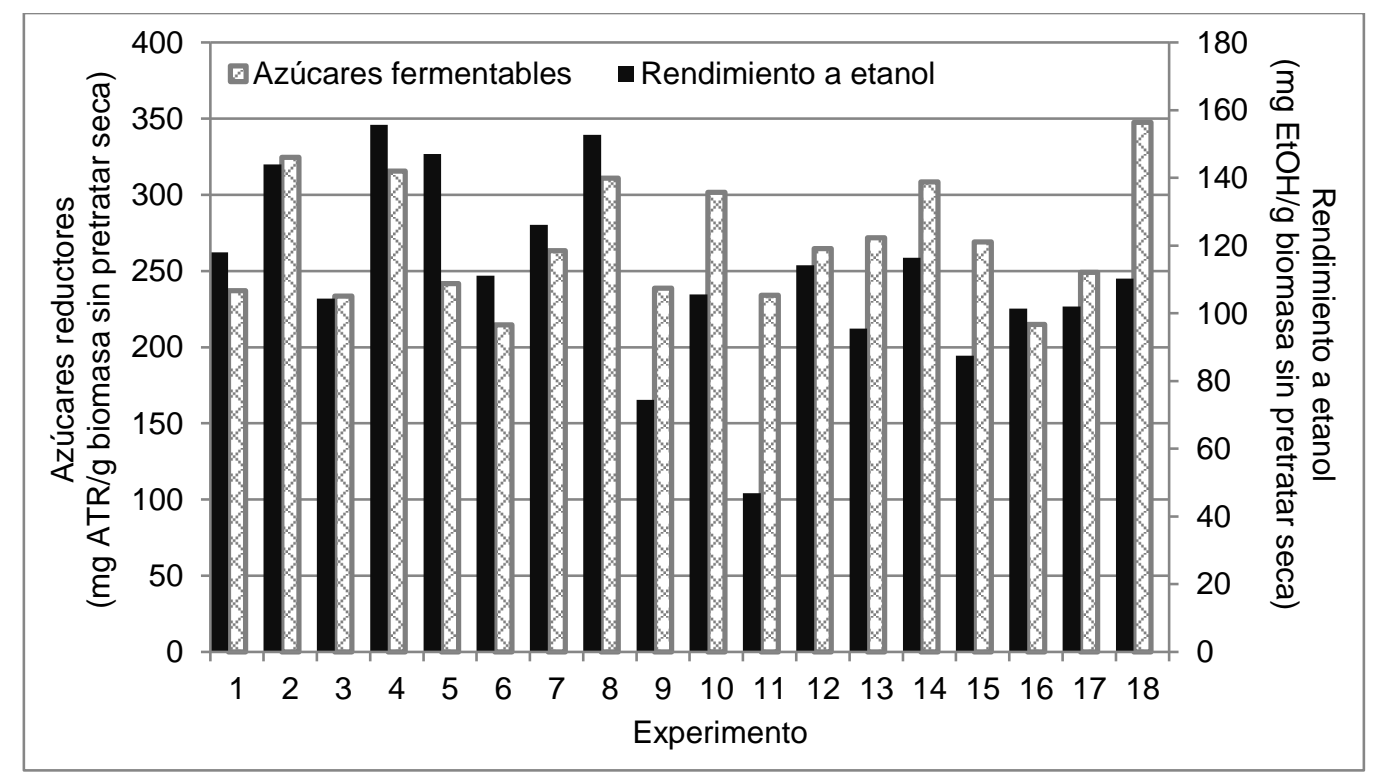

Fig. 4. Rendimiento de azúcares reductores totales (mg ATR/g biomasa sin pretratar seca) y rendimiento de etanol ( $\mathrm{mg} \mathrm{EtOH} / \mathrm{g}$ biomasa sin pretratar seca) de cada uno de los experimentos del pretratamiento deslignificación alcalina

La Tabla 9 muestra los resultados del diseño experimental llevado a cabo para hallar las mejores condiciones de operación para el pretratamiento deslignificación alcalina. Los resultados finales permiten determinar que es posible producir $155,69 \mathrm{mg}$ de etanol/g biomasa seca sin pretratar (correspondiente a 197,33 litros etanol/ton biomasa seca alimentada) a partir la fracción sólida obtenida del pretratamiento con $\mathrm{NaOH}$ del Buchón de Agua (fermentación SSF). La hidrólisis de la fracción sólida producto del pretratamiento alcalino produjo asimismo $347,48 \mathrm{mg}$ de ATR/g de biomasa después de 30 horas.

Tabla 9. Resultados del diseño experimental

\begin{tabular}{|c|c|c|c|c|c|c|}
\hline Factor & Bajo & Alto & Óptimo & Respuesta & Óptimo & Validado \\
\hline Temperatura $\left({ }^{\circ} \mathrm{C}\right)$ & 66,36 & 133,64 & 131,54 & Etanol $(\mathrm{g} / \mathrm{L})$ & 31,10 & 29,29 \\
\hline Tiempo (min) & 0,32 & 3,68 & 212 & ATR $(g / L)$ & 47,16 & 49,60 \\
\hline$[\mathrm{NaOH}]$ (\%peso) & 0,66 & 2,34 & 0,96 & & & \\
\hline Relación sólido líquido (g/g) & 13,30 & 21,70 & 19,96 & & & \\
\hline
\end{tabular}




\section{DISCUSIÓN FINAL}

Con el desarrollo de la presente investigación se pudo establecer un proceso viable técnicamente para la producción de etanol a partir de Buchón de Agua proveniente de los embalses de E.P.M. A continuación se desglosan los principales hallazgos relacionados: 1. La producción de etanol mediante el sistema de Sacarificación y Fermentación Simultáneas (SSF, 9 horas de fermentación) con los sólidos obtenidos de los pretratamientos deslignificacion alcalina, ácido diluido, remojo con amoníaco y el peróxido alcalino presentó rendimientos de $71,34 \mathrm{mg}$ etanol/g biomasa seca sin pretratar $(12,51 \mathrm{~g} / \mathrm{L})$, de $57,03 \mathrm{mg}$ etanol $/ \mathrm{g}$ biomasa seca sin pretratar $(8,41 \mathrm{~g} / \mathrm{L}), 53,59 \mathrm{mg}$ etanol/g biomasa seca sin pretratar $(9,96 \mathrm{~g} / \mathrm{L})$ y $11,21 \mathrm{mg}$ etanol/g biomasa seca sin pretratar $(1,35 \mathrm{~g} / \mathrm{L})$. 2. Se evidenció que el pretratamiento con ácido diluido permite recuperar una gran cantidad de la celulosa en la fracción sólida, determinándose, a las condiciones del presente trabajo un rendimiento máximo de etanol de 190,35 litros/ton de biomasa fresca alimentada (seca). 3. Con las condiciones del diseño del pretratamiento Deslignificación alcalina se alcanzó un rendimiento de etanol de 197,33 litros/ton de biomasa fresca alimentada (seca). 4. Finalmente, es importante aclarar, que la producción de etanol con una materia prima no convencional, como la biomasa de Buchón de Agua, tiene un futuro promisorio, tomando como base la evidencia científica que deja la presente investigación, a través de la cual se pueden establecer rutas seguras para el proceso. Evidentemente, la implementación real del mismo está sujeta a ajustes técnicos asociados al escalado y la administración de los recursos.

\section{CONCLUSIONES}

De acuerdo al trabajo presentado y a los resultados obtenidos, se pueden plantear las siguientes conclusiones principales:

1. El Buchón de Agua presenta una composición en carbohidratos de más del $50 \%$, lo que indica que más de la mitad de la composición en peso seco del material consiste en azúcares y que dependiendo de la selección de la técnica de pretratamiento y del microrganismo utilizado, el material es susceptible de ser fermentado.

2. El pretratamiento con Ácido diluido permite recuperar una gran cantidad de la celulosa contenida en la biomasa del Buchón de Agua en la fracción sólida a condiciones de operación relativamente moderadas lo cual lo convierte en una técnica de pretratamiento promisoria para la producción de bioetanol.

3. El pretratamiento Deslignificación alcalina permite obtener el mayor rendimiento de etanol lignocelulósico con base en la biomasa seca alimentada al proceso, sin embargo, es un proceso que se lleva a cabo a condiciones de operación más severas por lo que los costos pueden ser un factor determinante a la hora de comparar su viabilidad respecto al pretratamiento de hidrólisis con Ácido diluido.

\section{AGRADECIMIENTOS}

Los autores agradecen al Programa Colombia Científica como fuente de financiación, en el marco de las convocatorias Ecosistema Científico, contrato No. FP44842- 218-2018.

\section{REFERENCIAS}

Abraham, M. y G. M. Kurup, Bioconversion of tapioca (Manihot esculenta) waste and water hyacinth (Eichhornia crassipes)-Influence of various physico-chemical factors, doi: 10.1016/0922-338X(96)88817-9, Journal of Fermentation and Bioengineering, 82(3), 259-263 (1996)

Aguirre, N. J., J. Palacio y J. J. Ramírez, Revista Ingenierías Universidad de Medellin. Revista Ingenierías Universidad de Medellín (Vol. 6). Universidad de Medellin (2007)

Ahn, D. J., S. K. Kim y H. S. Yun, Optimization of pretreatment and saccharification for the production of bioethanol from water hyacinth by Saccharomyces cerevisiae, doi:10.1007/s00449-011-0600-5, Bioprocess and Biosystems Engineering, 35(1-2), 35-41 (2012)

Aswathy, U. S., R. K. Sukumaran y otros cuatro autores, Bio-ethanol from water hyacinth biomass: An evaluation of enzymatic saccharification strategy, doi: 10.1016/J.BIORTECH.2009.08.019, Bioresource Technology, 101(3), 925-930 (2010)

Bhattacharya, A. y P. Kumar, Water hyacinth as a potential biofuel crop. Electronic Journal of Environmental, Agricultural and Food Chemistry, 9(1), 112-122 (2002)

Cheng, K.-K., B. Y. Cai y otros cinco autores, Sugarcane bagasse hemicellulose hydrolysate for ethanol production by acid recovery process, doi: 10.1016/J.BEJ.2007.07.012, Biochemical Engineering Journal, 38(1), 105-109 (2008)

EPM, Informe sostenibilidad 2009. Medellín (2009). 
Ganguly, A. y N. Saha, Effect of Alkali Pretreatment on Water Hyacinth Biomass for Production of Ethanol. Advanced Chemical Engineering Research, 2(2), 40-44 (2013)

Guragain, Y. N., J. De Coninck y otros tres autores, Comparison of some new pretreatment methods for second generation bioethanol production from wheat straw and water hyacinth, doi: 10.1016/J.BIORTECH.2010.11.125, Bioresource Technology, 102(6), 4416-4424 (2011)

Isarankura-Na-Ayudhya, C., T. Kongpanpee, T. Tantimongcolwat y P. Prabkate, Appropriate Technology for the Bioconversion of Water Hyacinth (Eichhornia crassipes) to Liquid Ethanol: Future Prospects for Community Strengthening and Sustainable Development. EXCLI Journal, 6, 167-176 (2007)

Kumar, A., L. K. Singh, y S. Ghosh, Bioconversion of lignocellulosic fraction of water-hyacinth (Eichhornia crassipes) hemicellulose acid hydrolysate to ethanol by Pichia stipitis, doi: 10.1016/j.biortech.2009.02.023Bioresource Technology, 100(13), 3293-3297 (2009)

Ma, F., N. Yang y otros cuatro autores, Combination of biological pretreatment with mild acid pretreatment for enzymatic hydrolysis and ethanol production from water hyacinth, doi: 10.1016/J.BIORTECH.2010.07.084, Bioresource Technology, 101(24), 9600-9604 (2010)

Masami, G., I. Usui y N. Urano, Ethanol production from the water hyacinth Eichhornia crassipes by yeast isolated from various hydrospheres. African Journal of Microbiology Research, 2(5), 110-113 (2008)

Mateus, L., O. Hernández, M. Velásquez, J. Díaz, Evaluación del pretratamiento con ácido sulfúrico diluido del pasto maralfalfa (Pennisetum glaucum x Pennisetum purpureum) para la producción de etanol, Revista Colombiana de Biotecnología (Vol. 14), Universidad Nacional de Colombia (2012)

Mishima, D., M. Tateda, M. Ike y M. Fujita, Comparative study on chemical pretreatments to accelerate enzymatic hydrolysis of aquatic macrophyte biomass used in water purification processes, doi: 10.1016/J.BIORTECH.2005.09.029, Bioresource Technology, 97(16), 2166-2172 (2006)

Nigam, J., Bioconversion of water-hyacinth (Eichhornia crassipes) hemicellulose acid hydrolysate to motor fuel ethanol by xylose-fermenting yeast, doi: 10.1016/S0168-1656(02)00013-5, Journal of Biotechnology, 97(2), 107-116 (2002)

Reales-Alfaro, J. G., L. T. Trujillo-Daza y otros tres autores, C T \& F : Ciencia, Tecnología y Futuro. CT\&F - Ciencia, Tecnología y Futuro (Vol. 5), Instituto Colombiano del Petróleo (ICP) - ECOPETROL S.A. (2013)

Singh, A. y N. R. Bishnoi, Comparative study of various pretreatment techniques for ethanol production from water hyacinth, doi: 10.1016/J.INDCROP.2012.11.026, Industrial Crops and Products, 44, 283-289 (2013)

Sluiter, A., B. Hames y otros cuatro autores, Determination of Ash in Biomass, doi: NREL/TP-510-42619, NREL, National Renewable Energy Laboratory (2005)

Sornvoraweat, B. y J. Kongkiattikajorn, Separated hydrolysis and fermentation of water hyacinth leaves for ethanol production, Asia-Pacific Journal of Science and Technology, 15(9), 794-802 (2010)

Wyman, C. E., V. Balan y otros seis autores, Comparative data on effects of leading pretreatments and enzyme loadings and formulations on sugar yields from different switchgrass sources, doi: 10.1016/J.BIORTECH.2011.06.069, Bioresource Technology, 102(24), 11052-11062 (2011)

Xia, A., J. Cheng y otros cuatro autores, Enhancing enzymatic saccharification of water hyacinth through microwave heating with dilute acid pretreatment for biomass energy utilization, doi: 10.1016/J.ENERGY.2013.09.019, Energy, 61, 158-166 (2013) 
